\title{
Variabilidade da frequência cardíaca em mulheres com hipermobilidade articular
}

\section{Heart rate variability in women with joint hipermobility}

Mariane de Oliveira Nunes ${ }^{1}$, Marcelo Custódio Rubira², Ana Paula Fernandes De Angelis Rubira³ ${ }^{3}$ Aline Cristina Pereira do Nascimento ${ }^{4}$, Alderico Rodrigues de Paula Júnior ${ }^{5}$, Rodrigo Alexis Lazo 0sório ${ }^{6}$

Estudo desenvolvido na

Faculdade São Lucas - Curso de Fisioterapia - Laboratório de Fisiologia Cardiovascular - Porto Velho (RO), Brasil.

${ }^{1}$ Mestre em Engenharia Biomédica; Docente da Faculdade São Lucas - Porto Velho (RO), Brasil.

2 Doutor em Ciências; Docente da Faculdade São Lucas - Porto Velho (RO), Brasil.

${ }^{3}$ Mestre em Odontologia; Docente da Faculdade São Lucas - Porto Velho (RO), Brasil.

${ }^{4}$ Discente do curso de PósGraduação em Bioengenharia Médica da Universidade do Vale do Paraíba (UNIVAP) - São José dos Campos (SP), Brasil.

${ }^{5}$ Doutor em Ciência da Computação; Docente da UNIVAP - São José dos Campos (SP), Brasil.

${ }^{6}$ Doutor em Ciências; Docente da UNIVAP - São José dos Campos (SP), Brasil.

ENDEREÇO PARA CORRESPONDÊNCIA

Marcelo C. Rubira - Av. Brasília, 3.062 - Bairro São Cristóvão - CEP: 76804-070 - Porto Velho (RO), Brasil - E-mail: marcelorubira@hotmail.com

\section{APRESENTAÇÃO}

jun. 2010

ACEITO PARA PUBLICAÇÃO mai. 2011

FONTE DE FINANCIAMENTO nenhuma

CONFLITO DE INTERESSES nada a declarar

Parecer de aprovação no Comitê de Ética n⿳2 230/08.
RESUMO: A hipermobilidade é a capacidade de desempenhar movimentos articulares com amplitude maior que o normal. A prevalência possui variações determinadas pela etnia, sexo, idade, atividade física e variações nos critérios de caracterização. Aproximadamente $30 \%$ dos adultos são portadores e apresentam feedback proprioceptivo, sensorial diminuído e espacial alterado da articulação levando a maior frequência de ativação e deformação dos mecanorreceptores nos músculos esqueléticos e na pele. $\mathrm{O}$ aumento dos impulsos aferentes dos mecanorreceptores sobre a área cardiovascular no bulbo altera o controle autonômico sobre o coração. O objetivo do estudo foi avaliar o balanço simpatovagal durante manobra de ortostatismo em mulheres com hipermobilidade. Participaram do estudo 27 voluntárias, com 19,97 $\pm 1,79$ anos, índice de massa corpórea abaixo de $25 \mathrm{~kg} / \mathrm{m}^{2}$, sedentárias e sem uso de medicação. Após diagnóstico da hipermobilidade articular, segundo o escore de Beighton, foram divididas em 2 grupos: 12 hipermóveis $(\mathrm{GH})$ e 15 não hipermóveis (GC). O eletrocardiograma foi realizado durante 10 minutos em supino e em pé para análise da variabilidade da frequência cardíaca. A banda de alta frequência (un) apresentou diminuição da atividade vagal no $\mathrm{GH}, \mathrm{p}<0,03$. O incremento de baixa frequência (un) foi maior no $\mathrm{GH}$ em relação ao GC, na manobra de ortostatismo, com aumento da atividade simpática, $p<0,03$. As voluntárias com hipermobilidade articular apresentaram resposta autonômica cardíaca alterada com hiporesponsividade vagal.

Descritores: instabilidade articular; células receptoras sensoriais; frequência cardíaca.

ABSTRACT : Joint hypermobility is the ability to make joint movements greater than normal. The prevalence has large variations determined by race, sex, age, physical activity and variations in characterization criteria. Approximately $30 \%$ of adults are considered carriers and present proprioceptive feedback and sensory decreased and joint space positioning altered leading to greater frequency of activation and deformation on the mechanoreceptors in the skeletal muscles and skin. The increase of afferent impulses of the receptors on the bulb cardiovascular area alters the autonomic control on the heart. The objective of the study was to evaluate sympathovagal balance during orthosthatic maneuver in women with hipermobility. Twenty-seven sedentary volunteers participated in this study, with mean age of $19.97 \pm 1.79$, body mass index below $25 \mathrm{~kg} / \mathrm{m}^{2}$ and without medication. After the joint hipermobility diagnosis according to Beighton score, they were divided into 2 groups: 12 with hipermobility (HG) and 15 without hipermobility (CG). The electrocardiogram was performed during 10 minutes at supine position and stand for analysis of heart rate variability. The band of high frequency (un) presented reduction in vagal activity in $\mathrm{HG}, \mathrm{p}<0.03$. The low frequency increment (un) was higher in HG when compared to CG in orthosthatic maneuver with increased sympathetic activity, $p<0.03$. The joint hypermobility volunteers presented autonomic cardiac response altered with low vagal responsiveness.

KeYwords: joint instability; sensory receptor cells; heart rate. 


\section{INTRODUCÃO}

A hipermobilidade articular (HA) é a capacidade de desempenhar movimentos articulares com amplitude maior que o normal ${ }^{1}$. Na HA, o feedback proprioceptivo e sensorial está diminuído e o posicionamento espacial alterado da articulação levam a maior frequência de ativação e deformação dos mecanorreceptores nos músculos e na pele $\mathrm{e}^{2}$. A principal causa da hipermobilidade é uma anormalidade na síntese de proteína do colágeno, resultando em tecido conjuntivo enfraquecido e distensível ${ }^{3}$. A frouxidão ligamentar predispõe à HA, subluxações, deslocamentos ocasionando alterações biomecânicas nas articulações ${ }^{3,4}$

Os mecanorreceptores convertem a energia mecânica da deformação física em potenciais de ação nervosos levando a um aumento na descarga aferente. Esses sinais aferentes fornecem informações sensoriais sobre as forças internas e externas que atuam na articulação, nos músculos e tendões ${ }^{5}$.

Segundo Coote e Bothams ${ }^{6}$ há dois tipos de fibras nervosas musculares aferentes que mostraram influenciar nas mudanças cardiovasculares: fibras do grupo III mecanicamente sensíveis e do grupo IV metabolicamente sensíveis.

Os mecanorreceptores musculares do tipo III possuem um limiar alto e tornam-se ativos em movimentos com maior amplitude, agindo reflexamente produzindo mecanismo de freio contra o superalongamento. Alterações que ocorrem nos músculos são informadas pelos fusos musculares, órgãos tendinosos de Golgi ${ }^{7}$ e pelas aferências de pequeno diâmetro para a medula espinhal, para regiões reticulares do tronco cerebral e para o córtex cerebral $^{8}$. Os impulsos aferentes provenientes de mecanorreceptores incidem sobre a área cardiovascular, alterando o controle autonômico sobre coração ${ }^{9}$.

Disfunções nas articulações afetam a modulação autonômica e a função cardíaca $^{10}$. A variabilidade da frequência (VFC) permite avaliar o balanço simpatovagal do coração de forma não invasiva, com alto grau de confiabilidade. Apesar da larga utilização da análise da VFC na compreensão dos fenômenos envolvidos com o sistema nervoso autônomo (SNA) em condições normais e patológicas, sua utilização na prática clínica ainda é escassa $^{11}$. A finalidade do estudo foi analisar o balanço simpatovagal, na manobra de ortostatismo, de mulheres com hipermobilidade e sua influência sobre a modulação do sistema nervoso autônomo.

\section{METODOLOGIA}

Estudo transversal, duplo cego, aprovado pelo Comitê de Ética em Pesquisa no 230/08 em 01/08/2008. As voluntárias foram informadas sobre a pesquisa e assinaram o termo de consentimento livre e esclarecido.

\section{Seleção da amostra}

Cento e cinquenta voluntárias foram examinadas no Laboratório de Reabilitação Cardiopulmonar em 2009 e vinte e sete foram mulheres incluídas no estudo.

As voluntárias responderam ao questionário com dados de identificação, antropométricos, morbidades, nível de atividade física, tabagismo, etilismo e uso de medicação. Os critérios de inclusão foram mulheres na faixa etária de 18 a 30 anos, que não realizavam nenhuma atividade física por pelo menos 10 minutos contínuos durante a semana $^{12}$, saudáveis, eletrocardiograma normal, colesterol total e frações, triglicérides dentro da normalidade de acordo com National Cholesterol Education Program ${ }^{13}$ e glicemia ${ }^{14}$ e com diagnóstico de HA. Foram excluídas portadoras de doenças cardiovasculares, com distúrbio osteomioarticular, dor, fumantes, em uso de medicamentos e com diagnóstico de síndrome da hipermobilidade articular.

As voluntárias foram acompanhadas durante dois meses e foram avaliadas na mesma fase do ciclo menstrual, na fase lútea.

Após as avaliações, foram diagnosticados 12 casos de hipermobilidade segundo critérios de Beigthon ${ }^{15}$ e 18 casos de síndrome da hipermobilidade articular segundo critérios de Brighton ${ }^{15}$.

A amostra foi divida em 2 grupos: 12 hipermóveis $(\mathrm{GH})$ e 15 não hipermóveis (GC).

O protocolo utilizado é apresentado na Figura 1.

\section{Diagnóstico da hipermobilidade articular}

O diagnóstico da HA foi baseado no sistema Beighton com pontuação máxima de nove pontos, considerando as manobras para o lado esquerdo e direito e a manobra de flexão anterior do tronco com apoio das duas mãos no chão com joelhos estendidos. Manobras do sistema Beighton: hiperextensão do quinto dedo igual ou maior que $90^{\circ}$, hiperextensão do cotovelo maior que $10^{\circ}$, hiperextensão do joelho maior que $10^{\circ}$, a posição do polegar para a face flexora do antebraço e flexão anterior do tronco com apoio das duas mãos no chão com joelhos estendidos $^{3}$. O diagnóstico da HA refere-se a um escore maior ou igual a quatro pontos.

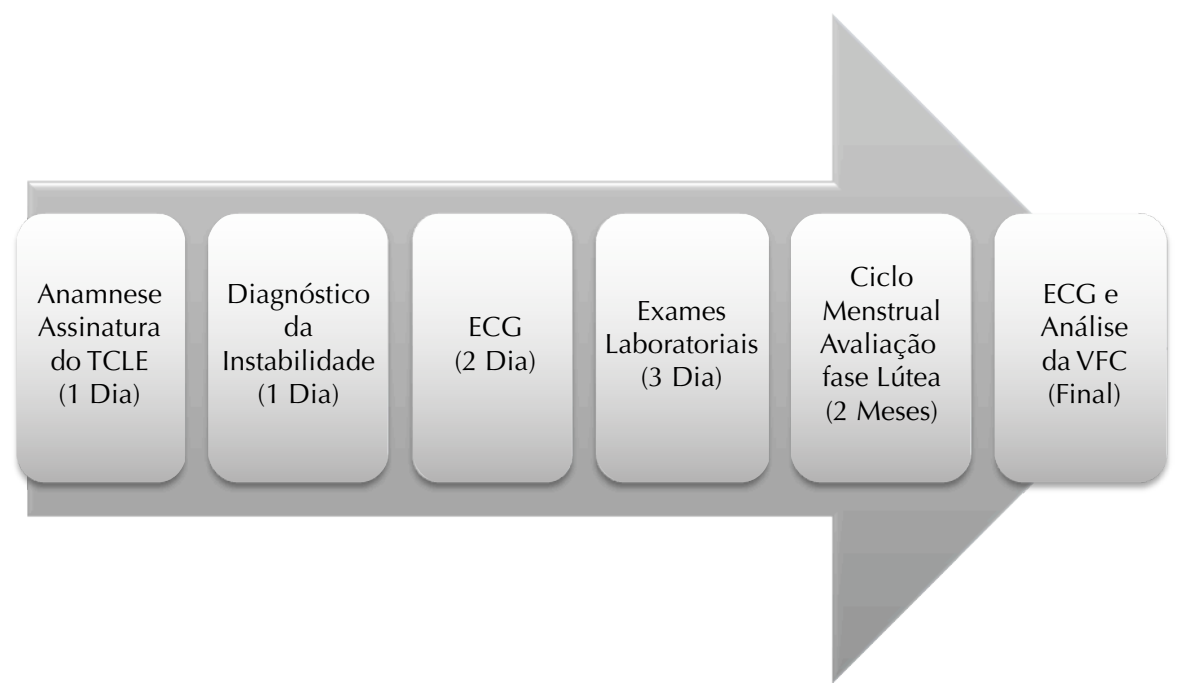

Figura 1. Protocolo utilizado para seleção da amostra 


\section{Diagnóstico da síndrome de hipermobilidade articular}

O diagnóstico da síndrome da HA foi baseado nos critérios revisados de Brighton, onde, além dos critérios de Beighton para avaliação da HA, são levados em consideração sintomas articulares e extra-articulares ${ }^{15,16}$.

\section{Eletrocardiograma}

As voluntárias permaneceram sentadas por 10 minutos em uma sala com temperatura ambiente entre 24 e $26^{\circ} \mathrm{C}$ para aferição dos sinais vitais.

O eletrocardiograma foi registrado na posição supina e os eletrodos foram fixados na região precordial para a capitação da derivação DII e obtenção dos intervalos entre duas ondas $R(R R)$.

Foi utilizado o eletrocardiógrafo digital (Nexus 10-System, software BIOTRACE, Mind Media B.V, Holanda) por 10 minutos na posição de supino e 10 minutos em pé. As voluntárias foram avaliadas na mesma fase do ciclo menstrual e no mesmo horário. Os sinais foram filtrados e convertidos para o formato de arquivo de texto.

\section{Análise espectral no domínio da frequência}

Após obtenção dos intervalos RR, Os dados foram analizados com Matlab 6.1 para realização da Transformada Wavelet Contínua (TWC) para o cálculo da área de baixa frequência $(B F-0,04-0,15)$ e da alta frequência ( $A F-0,15-0,4 \mathrm{~Hz})$.

BF representa a área simpática e parassimpática e AF corresponde à área parassimpática. O balanço autonômico foi dado pela razão entre a área simpática e parassimpática ${ }^{17}$.

A TWC foi calculada através do algorítmo "Morlet" desenvolvido e adaptado pelo Instituto de Pesquisa e Desenvolvimento da Universidade do Vale do Paraíba.

TWC é simultaneamente descrita, tanto no domínio da frequência quanto no domínio do tempo. Na análise de Wavelet utiliza-se a técnica de janelamento que possui tamanhos varáveis. Os espectogramas baseados em wavelets foram obtidos tomando o módulo da TWC e elevando-o ao quadrado ${ }^{18}$.

\section{Perfil bioquímico das voluntárias}

A coleta de sangue foi realizada das 7 às 8 horas no laboratório de análises clínicas, após um período de 10-12 horas de jejum. Foram analisadas as quantidades de colesterol total (CT) e triglicerídeos plasmáticos (TG) pelo teste enzimático colorimétrico com fator clareance de lipídios (LCF), lipoproteínas de alta densidade (HDLc) pelo método enzimático colorimétrico, lipoproteínas de baixa densidade (LDL-c) e lipoproteínas de muito baixa densidade (VLDL-c) pela equação de Friedwald e glicemia com método enzimático colorimétrico sem desproteinização.

\section{Análise estatística}

A análise estatística foi realizada com Sigma Stat 3.0. A amostra dos grupos controle e hipermóveis foi calculada a partir de estudo piloto e estimativa da amostra pela diferença média esperada e desvio padrão esperado com alfa de 0,05. Os dados apresentaram distribuição normal pelo teste Kolmogorov-Smirnov. Para a comparação da posição supina e em pé intragrupo foi utilizado o teste $t$ de Student pareado.

Com relação a comparação da posição supina e em pé intergrupo utilizou-se o Teste $t$ de Student não pareado. Todas as discussões no presente trabalho foram realizadas no nível de 0,05 de significância.

\section{RESULTADOS}

Participaram 27 jovens, do gênero feminino, com 19,97 $\pm 1,79$ anos e com valores médios de índice de massa corpórea (IMC), colesterol e frações e glicemia dentro da faixa considerada ideal ${ }^{13,14}$. A média e desvio padrão dos valores obtidos de peso, altura, IMC, colesterol e frações e glicemia estão expressos na Tabela 1.

\section{Diagnóstico da hipermobilidade articular}

A pontuação para o diagnóstico da HA do $\mathrm{GH}$ apresentou diferença estatisticamente significante $(p<0,01)$ com média de 6,86 $\pm 0,95$ e as não hipermóveis de $1,43 \pm 0,85$ pontos, de acordo com os critérios de Beighton.

\section{Respostas da variabilidade da frequência cardíaca}

A banda de BF (un) aumentou significativamente e houve uma diminuição significativa da banda de AF (un), resposta fisiológica durante a manobra de ortostatismo, em ambos os grupos.

Ao compararmos os momentos dos espectros BF (un) e AF (un), em supino e em pé do GC com as do $\mathrm{GH}$, não observou-se diferença significante conforme dados apresentados na Tabela 2 .

Com relação ao balanço simpatovagal, não houve diferença significante entre os momentos supino do grupo controle com as hipermóveis e em pé do controle com as hipermóveis.

Durante a comparação do incremento da resposta de BF e diminuição das respostas de AF, na manobra de

Tabela 1. Comparação das características demográficas e clínicas das voluntárias do grupo controle e do grupo de hipermóveis

\begin{tabular}{lccc}
\hline & $\mathrm{GH}(\mathrm{n}=12)$ & $\mathrm{GC}(\mathrm{n}=15)$ & Valor $\mathrm{p}$ \\
\hline Massa corporal $(\mathrm{kg})$ & $63,95 \pm 7,42$ & $64,85 \pm 5,12$ & $0,63^{*}$ \\
Estatura $(\mathrm{m})$ & $1,62 \pm 0,05$ & $1,61 \pm 0,07$ & $0,80^{*}$ \\
$\mathrm{IMC}\left(\mathrm{kg} / \mathrm{m}^{2}\right)$ & $19,73 \pm 2,74$ & $20,13 \pm 2,64$ & $0,83^{*}$ \\
$\mathrm{CT}(\mathrm{mg} / \mathrm{dL})$ & $157,78 \pm 30,13$ & $160,95 \pm 31,39$ & $0,80^{*}$ \\
HDL-c $(\mathrm{mg} / \mathrm{dL})$ & $52,6 \pm 13,5$ & $48,2 \pm 8,5$ & $0,50^{*}$ \\
VLDL-c $(\mathrm{mg} / \mathrm{dL})$ & $12,58 \pm 4,9$ & $12,61 \pm 4,75$ & $0,98^{*}$ \\
LDL-c $(\mathrm{mg} / \mathrm{dL})$ & $92,60 \pm 23,23$ & $100,04 \pm 28,13$ & $0,30^{*}$ \\
TG $(\mathrm{mg} / \mathrm{dL})$ & $62,9 \pm 24,63$ & $63,09 \pm 23,79$ & $0,98^{*}$ \\
Glicemia(mg/dL) & $85,20 \pm 10,02$ & $82,14 \pm 11,00$ & $0,85^{*}$ \\
\hline
\end{tabular}

Valores expressos em média e desvio padrão; *poder teste >0,50; GH: grupo de hipermóveis; GC: grupo controle; HDL-c: lipoproteínas de alta densidade; LDL-c: lipoproteínas de baixa densidade; VLDL-c: lipoproteínas de muito baixa densidade; TG: triglicerídeos; CT: colesterol total; IMC: índice de massa corpórea 
Tabela 2. Comparação do índice de BF e AF (un) dos intervalos entre duas ondas $R$ do eletrocardiograma (RR) entre os momentos supino e em pé, e a resposta de mudança de decúbito, de supino para em pé, do grupo controle e do grupo de hipermóveis, respectivamente.

\begin{tabular}{|c|c|c|c|}
\hline & Supino & Em Pé & Valor $\mathrm{p}$ \\
\hline \multicolumn{4}{|c|}{ BF (un) } \\
\hline $\mathrm{GH}$ & $35,8 \pm 9,4$ & $71,8 \pm 11,7$ & $0,001^{*}$ \\
\hline GC & $42,2 \pm 9,5^{\ddagger}$ & $69,4 \pm 10,5^{\ddagger}$ & $0,001^{*}$ \\
\hline \multicolumn{4}{|c|}{ AF (un) } \\
\hline $\mathrm{GH}$ & $64,1 \pm 9,4$ & $28,1 \pm 11,7$ & $0,001^{*}$ \\
\hline GC & $57,7 \pm 9,6^{+}$ & $30,5 \pm 10,5^{+}$ & $0,001^{*}$ \\
\hline \multicolumn{4}{|l|}{$\mathrm{BF} / \mathrm{AF}$} \\
\hline $\mathrm{GH}$ & $0,72 \pm 0,58$ & $3,26 \pm 2,58$ & $0,001^{*}$ \\
\hline GC & $0,78 \pm 0,33^{\#}$ & $2,77 \pm 1,7^{\#}$ & $0,001^{*}$ \\
\hline
\end{tabular}

$\mathrm{GH}$ : grupo hipermóveis; GC: grupo controle; BF: baixa frequência; AF: alta frequência. ${ }^{\ddagger} p>0,05$ comparação da resposta $B F(u n),{ }^{\dagger} p>0,05$ comparação da resposta $A F(u n)$ e ${ }^{\#} p>0,05$ comparação do balanço BF/AF no momento supino e no momento em pé entre GH e o GC.

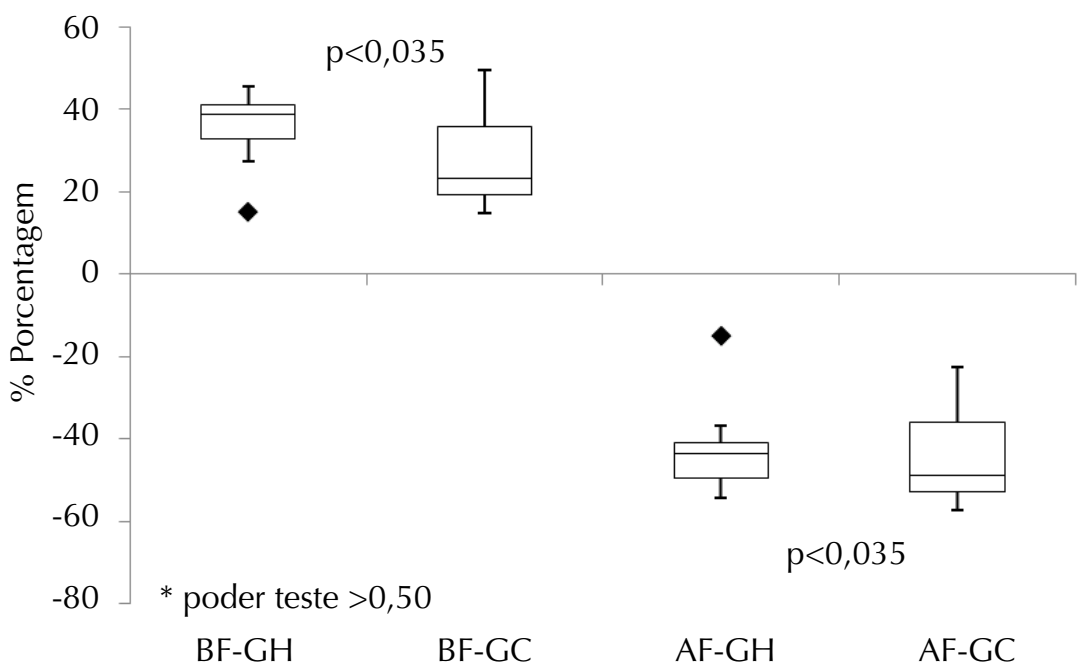

Figura 2. Comparação do incremento da resposta de baixa frequência (BF) e diminuição da resposta de alta frequência (AF) na variabilidade de frequência cardíaca durante manobra de ortostatismo no grupo hipermóvel $(\mathrm{GH})$ e grupo controle $(\mathrm{GC})$

ortostatismo, observou-se uma maior diminuição de AF nas hipermóveis comparado ao grupo controle conforme mostrado na Figura 2.

\section{DISCUSSÃO}

Nesse estudo obteve-se como resultados mais importantes: 1) diferença de resposta simpática e parassimpática, diferença significante entre o incremento da resposta de baixa frequência e diminuição da resposta de alta frequência durante a manobra de ortostatismo no grupo de hipermóveis.

O IMC, colesterol total e frações e glicemia das voluntárias apresentaram-se dentro dos parâmetros de normalidade, uma vez que são conhecidos os efeitos da obesidade, glicemia, colesterol e frações no sistema cardiovascular ${ }^{13,14}$.

Estudos demonstram que tanto a contração, como o alongamento do músculo esquelético, ativa fibras nervosas aferentes, no indivíduo normal, ocasionando um aumento da frequência cardíaca (FC) por diminuição do tônus vagal cardíaco, levando assim a uma diminuição da VFC ${ }^{19}$.

Acredita-se que os mecanorreceptores musculares do grupo III estejam mais ativados em pacientes com diagnóstico de $\mathrm{HA}^{4}$.

Os estudos de Gladwell e Coote ${ }^{20}$ e de Gladewell et al. ${ }^{9}$ sugeriram que a diminuição da VFC, durante o alongamento muscular, foi devido à ativação do mecanorreceptor do grupo III e que o estímulo aferente produziu aumento da FC pela inibição do tônus vagal. Em nosso estudo, as hipermóveis apresentaram resposta diminuída do parassimpático na manobra de ortostatismo.

Budgell e Polus ${ }^{21}$ relatam que disfunções nas articulações torácicas afetam a modulação autonômica e a função cardíaca.

A modulação autonômica é o mecanismo responsável pelo controle da FC em voluntários normais. Na posição de supino, os sistemas simpático e parassimpático são tonicamente ativos, com um efeito vagal predominante. A variação da FC observada pelo intervalo entre duas ondas $\mathrm{R}$ do eletrocardiograma, pode fornecer informações importantes sobre a integridade da função neurocardíaca ${ }^{22}$.

Estudos têm demonstrado que a diminuição da VFC está relacionada a um maior índice de morbidade e mortalidade cardiovascular. Por essas razões, utilizam-se manobras respiratórias, mudanças de posição e bloqueios farmacológicos do SNA, na tentativa de investigar a VFC ${ }^{23}$. 
Paschoal et al. ${ }^{24}$ relatam que durante a mudança postural em indivíduos de diferentes faixas etárias ocorrem variações no tônus simpatovagal, como aumento da atividade simpática e retirada vagal. Nosso estudo utilizou a manobra de ortostatismo, manobra fisiológica, para a análise da VFC.

Em estudo realizado com adolescentes e adultos jovens sadios, verificou-se que durante os testes de inclinação houve uma importante elevação dos componentes de baixa frequência e discreto decréscimo nos componentes de alta frequência. Esses dados sugeriram que o aumento da FC observado com a inclinação é mediado tanto pela retirada vagal como pelo aumento da atividade simpática ${ }^{25}$.

Lindqvist ${ }^{26}$, em sua pesquisa sobre os efeitos da postura na oscilação do sistema cardiovascular, observou que a VFC foi maior na posição supina, mas que a simples alteração para a postura em pé produzia uma diminuição desta variabilidade.
Os resultados do estudo demonstram que os padrões de resposta da FC das voluntárias estudadas, durante manobra de ortostatismo, estão de acordo com os dados encontrados na literatura.

Bevegard e Shepherd ${ }^{27}$ evidenciaram que na posição supina, o volume de ejeção em supino é quase máximo e que, em contraste à força de gravidade na posição ereta, age contrariando o fluxo de retorno para o coração, resultando em volume de ejeção reduzido e FC aumentada.

A redução da VFC tem sido apontada como um forte indicador de risco relacionado a eventos adversos em indivíduos normais e em pacientes com um grande número de doenças, refletindo o papel vital que o SNA desempenha na manutenção da saúde ${ }^{28}$.

A elevação da modulação parassimpática induz uma estabilidade elétrica do coração, ao passo que a atividade simpática elevada aumenta a vulnerabilidade e o risco de eventos cardiovasculares ${ }^{29}$.
Em relação ao AF (un), observou-se uma diminuição maior da atividade no GH comparado ao GC, o que nos revela uma hipoatividade vagal e uma diminuição da VFC frente a mudança postural.

Dessa forma, hipermóveis necessitam de enfoque terapêutico diferenciado quanto à prescrição de exercício físico de fortalecimento e propriocepção e maior acompanhamento na prática regular de atividade física.

É importante destacar que uma das limitações do presente estudo está relacionada à amostra reduzida, assim como os métodos utilizados para avaliar a mobilidade articular em diversas condições de saúde serem considerados limitados.

CONCLUSÃO

Esses resultados sugerem que a hipermobilidade articular em mulheres jovens está associada a uma resposta autonômica cardíaca alterada com hiporresponsividade vagal.

\section{REFERÊNCIAS}

1. Ishaq M, Sheikh I, Kumar A, Chand S. Joint laxity and hypermobility in adults at an industrial area of Karachi. J Coll Physicians Surg Pak. 2010;20(11):753-6.

2. Mallik AK, Ferrell WR, McDonald AG, Sturrock RD. Impaired proprioceptive acuity at the proximal interphalangeal joint in patients with the hypermobility syndrome. Br J Rheumatol. 1994;33(7):631-7.

3. Hakim AJ, Sahota A. Joint hypermobility and skin elasticity: the hereditary disorders of connective tissue. Clin Dermatol. 2006;24(6):521-33.

4. Smith R, Damodaran AK, Swaminathan S, Campbell R, Barnsley L. Hypermobility and sports injuries in junior netball players. Br J Sports Med. 2005;39(9):628-31.

5. Lephart SM. Como restabelecer o controle neuromuscular. In: Prentice WE, editor. Técnicas de reabilitação em medicina esportiva. Barueri: Manole; 2002. p. 88-106.

6. Coote JH, Bothams VF. Cardiac vagal control before, during and after exercise. Exp Physiol. 2001;86(6):811-5.

7. Bergenheim M, Johansson H, Pedersen J, Ohberg F, Sjolander P. Ensemble coding of muscle stretches in afferent populations containing different types of muscle afferents. Brain Res. 1996;734(1-2):157-66.
8. Fisher JP, White MJ. Muscle afferent contributions to the cardiovascular response to isometric exercise. Exp Physiol. 2004;89(6):639-46.

9. Gladwell VF, Fletcher J, Patel N, Elvidge LJ, Lloyd D, Chowdhary $\mathrm{S}$, et al. The influence of small fibre muscle mechanoreceptors on the cardiac vagus in humans. J Physiol. 2005;567(Pt 2):713-21.

10. Eingorn AM, Muhs GJ. Rationale for assessing the effects of manipulative therapy on autonomic tone by analysis of heart rate variability. J Manipulative Physiol Ther. 1999;22(3):161-5.

11. Vanderlei LC, Pastre CM, Hoshi RA, Carvalho TD, Godoy MF. Basic notions of heart rate variability and its clinical applicability. Rev Bras Cir Cardiovasc. 2009;24(2):205-17.

12. Pardini R MS, Araújo T, Matsudo V, Andrade E, Braggion G, Andrade D, et al. Validação do questionário internacional de nível de atividade física (IPAQ - versão 6): estudo piloto em adultos jovens brasileiros. Rev Bras Ciên Mov. 2001;9(3):45-52.

13. Drown DJ, Engler MM. New guidelines for blood cholesterol by the National Cholesterol Education Program (NCEP). National Cholesterol Education Program (NCEP). Prog Cardiovasc Nurs. 1994;9(1):43-4. 


\section{Referências (cont.)}

14. Chobanian AV, Bakris GL, Black HR, Cushman WC, Green LA, Izzo Jr JL, et al. Seventh report of the Joint National Committee on Prevention, Detection, Evaluation, and Treatment of High Blood Pressure. Hypertension. 2003;42(6):1206-52.

15. Hakim A, Grahame R. Joint hypermobility. Best Pract Res Clin Rheumatol. 2003;17(6):989-1004.

16. Nijs J, Aerts A, De Meirleir K. Generalized joint hypermobility is more common in chronic fatigue syndrome than in healthy control subjects. J Manipulative Physiol Ther. 2006;29(1):32-9.

17. Longo A, Ferreira D, Correia MJ. [Variability of heart rate]. Rev Port Cardiol. 1995;14(3):241-62, 190.

18. Burri H, Chevalier P, Arzi M, Rubel P, Kirkorian G, Touboul P. Wavelet transform for analysis of heart rate variability preceding ventricular arrhythmias in patients with ischemic heart disease. Int J Cardiol. 2006;109(1):101-7.

19. al-Ani M, Robins K, al-Khalidi AH, Vaile J, Townend J, Coote $\mathrm{JH}$. Isometric contraction of arm flexor muscles as a method of evaluating cardiac vagal tone in man. Clin Sci (Lond). 1997;92(2):175-80.

20. Gladwell VF, Coote JH. Heart rate at the onset of muscle contraction and during passive muscle stretch in humans: a role for mechanoreceptors. J Physiol. 2002;540(Pt 3):1095-102.

21. Budgell B, Polus B. The effects of thoracic manipulation on heart rate variability: a controlled crossover trial. J Manipulative Physiol Ther. 2006;29(8):603-10.
22. Ribeiro JP, Moraes Filho, RS. Variabilidade da frequência cardíaca como instrumento de investigação do sistema nervoso autônomo. Rev Bras Hipertens. 2005;12(1):14-20.

23. Alonso DO, Forjaz CL, Rezende LO, Braga AM, Barretto $A C$, Negrao CE, et al. [Heart rate response and its variability during different phases of maximal graded exercise]. Arq Bras Cardiol. 1998;71(6):787-92.

24. Paschoal MA, Volanti M, Pires CS, Fernandes FC. Variabilidade da frequência cardíaca em diferentes faixas etárias. Rev Bras Fisioter. 2006;10(4):413-9.

25. Ribeiro MP, Brum JM, Ferrario CM. [A spectral analysis of the heart rate. The basic concepts and their clinical application]. Arq Bras Cardiol. 1992;59(2):141-9.

26. Lindqvist A. Noninvasive methods to study autonomic nervous control of circulation. Acta Physiol Scand Suppl. 1990;588:1-107.

27. Bevegard BS, Shepherd JT. Regulation of the circulation during exercise in man. Physiol Rev. 1967;47(2):178-213.

28. Pumprla J, Howorka K, Groves D, Chester M, Nolan J. Functional assessment of heart rate variability: physiological basis and practical applications. Int J Cardiol. 2002;84(1):1-14.

29. Brunetto AF, Silva BM, Roseguini BT, Hirai DM, Guedes DP. Limiar ventilatório e variabilidade de frequência cardíaca em adolescentes. Rev Bras Med Esporte. 2005; 11(1):22-7. 